\title{
Matching Testing Possibilities and Needed Experiments for Successful Vapor Compression Cycles in Microgravity
}

\author{
Leon P. M. Brendel, a), James E. Braun,, b), \\ Eckhard A. Groll, ${ }^{1, \mathrm{c})}$
}

${ }^{1}$ Ray W. Herrick Laboratories, School of Mechanical Engineering, Purdue

University, West Lafayette, 47907, USA

a) Corresponding author: brendel@purdue.edu

b) jbraun@purdue.edu

c) groll@purdue.edu

\begin{abstract}
The literature shows five decades of interest in vapor compression cooling for spacecraft, while only very few conclusions can be drawn because of either nonsystematic approaches or sparsely documented experiments. In contrast, the demand for high COP refrigerators and freezers has increased with the emerging plans of long duration manned space travel. Research is needed exploiting all available testing approaches to investigate two-phase cycles in microgravity environments. This paper presents relevant testing possibilities with their characteristics and outlines open questions regarding vapor compression cycles in space. Beneficial experiments are derived from open questions and matched with available testing methods to prescribe a path towards reliable and efficient refrigeration systems in microgravity.
\end{abstract}

\section{INTRODUCTION}

One of the first references to microgravity refrigeration [1] points out the need for cooling on spacecraft and suggests a modified absorption chiller to operate despite the absence of gravity in 1967. Ten years later, a report about the biomedical results from SKYLAB, the United States' first space station, mentions that certain urine samples could not be analyzed properly due to the lack of refrigeration and include a refrigeration ability for the return vehicle in the final recommendations [2]. Generally, storing biological samples is among the main reasons for refrigeration in microgravity. However, in recent years, scientific evidence underlines an additional argument. Manned mission will become multiple years long during which astronauts need an acceptable food quality [3], which in return may require refrigeration, because astronaut food in shelf storage is subject to nutrition degradation with time [4].

Refrigeration on spacecraft could be realized using the Sterling cycle, the reversed Brayton cycle or the vapor compression cycle. While the first two have the benefit of pure single-phase gas flow, the vapor compression cycle could operate with a higher COP and thereby reduce the power weight penalty. Despite this advantage, 
only five attempts of vapor compression cooling in microgravity have been documented ([5],[6],[7],[8]). Not only is the number very small, but the documents rarely discuss encountered challenges in detail. Another remarkable observation is that the five vapor compression cycles in microgravity were exclusively onboard orbiting spacecraft, which is an expensive testing platform. Researchers in related fields, such as two-phase flow heat transfer or heat pipes utilize not only orbiting spacecraft but also inclined test stands, parabolic flights and sounding rockets (examples can be found in [9],[10] and [11], respectively, but many more exist), all of which are generally less expensive experiments than testing on an orbital spacecraft. The variety of testing methods emerges from the fundamental problem, that gravity cannot be shielded so that various kinds of free fall are the only possibility to perform microgravity testing where earth's gravity field is sensible. The testing methods are specifically drop towers, parabolic flights, sounding rockets (suborbital) and orbital spacecraft. Despite not simulating the absence of gravity, testing on inclined stands is used as a low cost access to understanding effects of gravity or verifying gravity independence.

This paper suggests that vapor compression cycle research should, just as related research fields do, also access as many different testing platforms as are useful. Therefore, this paper puts forward a comprehensive list of testing possibilities and needed experiments to point out compatibilities.

\section{MICROGRAVITY TESTING POSSIBILITIES}

Every researcher concerned with microgravity, faces the problem that gravity cannot be shielded off in earth surface experiments. In addition, the magnitude of gravity decreases only slowly with distance from earth. For example, the magnitude of gravity at an altitude of $400 \mathrm{~km}$, approximately the orbit of the International Space Station (ISS), is still $90 \%$ of the gravity magnitude experienced on the surface of earth. To perform microgravity experiments, there are four testing possibilities available, all of which essentially rely on free fall. Apart from those, also testing at varying inclinations can reveal effects of gravity or gravity independence. Although tilting a setup can never simulate microgravity, it is a very relevant experiment and therefore included in the discussions in this paper. The following sections introduce the testing possibilities in general in the order of achievable microgravity testing time. Table 1 provides specific examples of microgravity testing platforms and their capabilities as well as cost.

\section{Inclinable Test Setup}

An inclinable test stand cannot simulate microgravity but allows to tilt an experimental setup. This yields information about the effects of gravity and/or verifies that a given setup operates gravity independent, for example if measurements do not vary significantly while changing the inclination angle. Inclinable test stands have the advantages of relatively low cost and easy accessibility. Researchers can modify the experiment and also intervene by manual 
operation of components, such as valves or switches, avoiding the need for automatic execution and control of the experiment. The authors know from experience that a structure to incline an approximately $200 \mathrm{~W}$ cooling capacity refrigeration test setup can be built for less than $\$ 10000$.

Drop Tower

Drop towers are the intuitive realization of free fall. Usually the experiment is inserted into a capsule, which falls through an evacuated space, thereby experiencing a high quality of microgravity. Advanced drop towers use a catapult to start the experiment from the ground, thereby doubling the time of experienced microgravity at a given height. This is for example implemented in the drop tower of ZARM in Bremen (Germany), which offers $9.3 \mathrm{~s}$ of microgravity testing [12], similar to $10 \mathrm{~s}$ possible in a $700 \mathrm{~m}$ tall drop-shaft without catapult at the Japanese Microgravity Center (JAMIC) [13].

\section{Parabolic Flights}

Parabolic flights achieve free fall by steering the airplane to fly a parabola. However, the quality of the microgravity depends on ambient wind conditions and the pilot flying closely aligned with the theoretical trajectory. The time of experienced free fall is $15 \mathrm{~s}$ to $30 \mathrm{~s}$ ([14]) and flight providers usually fly 20 to 40 parabolas consecutively. In between the parabolas, the experienced gravity is $1.8 \mathrm{~g}$ and may be used for hyper gravity experiments. Airplanes can also fly on a trajectory that simulates Lunar or Martian gravity levels. During parabolic flights, researchers can be next to their experiment to modify it or intervene.

\section{Sounding Rockets}

Sometimes also called suborbital flights, sounding rockets are characterized by reaching an altitude of at least $100 \mathrm{~km}$ but not orbit around earth. A capsule housing all payloads is decoupled from the carrier rocket during the flight and experiences several minutes of weightlessness during free fall. The experiment is typically stored in an enclosure and no humans travel in the capsule. All processes must therefore be automated. For this reason, the capsule provides electric power as well as a variety of digital signals, among them an indicator of the start of the microgravity phase of the flight. Researchers frequently use cameras to capture results of their experiments. It is important to distinguish between traditional and commercial flight opportunities. The former can reach more than 10 minutes of zerogravity testing time [13], while typical commercial providers offer only 3 minutes but at a much lower cost [15].

\section{Orbital spacecraft}

A supply spacecraft, lower earth orbit satellites, or the ISS spend days, months and years in space. Although they are still in the field of earth gravity, the 
experienced weightlessness is of high quality due to the constant free fall while orbiting. The main disadvantage of experiments on orbital spacecraft is the long lead time and high cost. Experiments should be mostly controlled remotely or be automated to avoid penalizing crew time. Experiments on orbital spacecraft should therefore have a high technology readiness level. Since access to large orbital spacecraft is very limited, [16] also points out recoverable satellites as good testing platforms that have been pursued by several countries in the past.

\begin{tabular}{|c|c|c|c|c|l|c|}
\hline Platform & $\mathbf{A t}_{\mathrm{e}}=\mathbf{0}$ & $\begin{array}{c}\text { Space/Vol } \\
\text { ume for } \\
\text { payload }\end{array}$ & $\begin{array}{c}\text { Mass } \\
\text { for } \\
\text { payload }\end{array}$ & $\begin{array}{c}\text { Cost in } \\
\text { US\$ }\end{array}$ & $\begin{array}{c}\text { Possibility for } \\
\text { manual } \\
\text { intervention }\end{array}$ & Ref \\
\hline $\begin{array}{c}\text { Inclined } \\
\text { stand }\end{array}$ & $\begin{array}{c}\text { Not } \\
\text { Applicable }\end{array}$ & $1.7 \mathrm{~m}^{3}$ & $100 \mathrm{~kg}$ & $\$ 10000$ & $\begin{array}{l}\text { High - } \\
\text { experiment } \\
\text { can be } \\
\text { manually } \\
\text { accessed at } \\
\text { any time }\end{array}$ & {$[17]$} \\
\hline $\begin{array}{c}\text { Drop } \\
\text { tower }\end{array}$ & $9.3 \mathrm{~s}$ & $0.34 \mathrm{~m}^{3}$ & $162 \mathrm{~kg}$ & $*$ & $\begin{array}{l}\text { Low - usually } \\
\text { impossible }\end{array}$ & {$[13]$} \\
\hline $\begin{array}{c}\text { Parabolic } \\
\text { flight }\end{array}$ & $20-30 \mathrm{~s}$ & $\begin{array}{c}\text { Area of } \\
\text { im }\end{array}$ & $* *$ & $\sim \$ 38500$ & $\begin{array}{l}\text { High - } \\
\text { researchers } \\
\text { can intervene } \\
\text { experiment }\end{array}$ & {$[18]$} \\
\hline $\begin{array}{c}\text { Commerc } \\
\text { ial } \\
\text { sounding } \\
\text { rocket }\end{array}$ & $3 \mathrm{~min}$ & $0.052 \mathrm{~m}^{3}$ & $11.3 \mathrm{~kg}$ & $\sim \$ 115000$ & $\begin{array}{l}\text { Low - usually } \\
\text { impossible }\end{array}$ & {$[15]$} \\
\hline $\begin{array}{c}\text { Orbital } \\
\text { spacecraft }\end{array}$ & $\begin{array}{l}\text { Several } \\
\text { months }\end{array}$ & $\sim 0.2 \mathrm{~m}^{3}$ & $\sim 165$ & N/A & $\begin{array}{l}\text { Low - usually } \\
\text { long term } \\
\text { experiments } \\
\text { should be } \\
\text { operated } \\
\text { remotely to } \\
\text { save crew time }\end{array}$ & {$[19]$} \\
\hline
\end{tabular}

Table 1. Examples of testing platforms. The information in the table provides specific examples and not limits or ranges for the testing platform characteristics. *Example payload to be inserted into ISS soon.

**Infoimation not available.

\section{NEEDED EXPERIMENTS REGARDING VCC IN ZERO-G AND CRITICALITY FOR SUCCESS}

\section{Open Questions and Needed Experiments}

The understanding of vapor compression systems in microgravity that is available in the literature is poor. Only five different vapor compression systems have microgravity flight experiment and none of them is documented well. The literature is therefore an insufficient source when asking for all the problems that a VCC in microgravity could have. The following is a description of potential 
problems and experiments that could verify a solution. The information is summarized in Table 2.

\section{Oil-Lubrication}

Most compressors rely on a gravity fed oil-sump to ensure proper lubrication. Without gravity, oil does not reliably return to the sump such that the compressor could be drained from oil. Two solutions are available: 1) Development of an oil-free compressor with the positive byproduct that any effect of oil onto the heat transfer would be removed. 2) Design of a compressor that solely relies on mist lubrication instead of an oil-sump. The gravity independence of the oil-lubrication should be verified by compressor performance testing at different compressor orientations.

\section{System Start-up}

Microgravity imposes an uncertainty on two-phase cycles: Where is the liquid phase of the refrigerant when the system is turned off? In microgravity, the liquid refrigerant is not collected at the lowest point of the system as it would be in terrestrial systems. Instead, its location is dictated by capillary forces or small maneuvers of the spacecraft. In a worst case scenario, the liquid will accumulate at the suction valve or in the compression chamber if the compressor does not have valves. Similarly, in a compressor without valves, the refrigerant could enter the compression chamber through the discharge port. It seems therefore necessary, to develop a design that ensures liquid cannot enter the compression chamber. The modification should allow the system start-up with liquid at various locations in the cycle. The compressor protection could be tested on an inclinable test stand, which places the compressor at the bottom of the cycle, such that a liquid column is pulled towards the compressor by gravity.

\section{System Operation}

When using an oil-free compressor, there is no apparent reason that the system operation would be significantly hindered by the absence of gravity. Steady-state microgravity testing should still be performed to increase the TRL of VCC in microgravity. Since there is no generally accepted correlation for heat transfer coefficients in microgravity, the data collected from the heat exchangers would have a high merit, too.

Because the gravity dependence of two-phase flow decreases with increased flow velocity, a vapor compression cycle could be designed to achieve gravity independent operation by using appropriate pipe sizing. [20] conducted a theoretical investigation of such a VCC and its problems.

\section{Dehumidification System}

After condensation of water-vapor from the air onto the evaporator coil or after a defrost procedure, the water usually drains driven by gravity for terrestrial applications. In microgravity, the water would not drain efficiently and could clog 
the evaporator fins or lead to a larger frost build up during operation. It is therefore necessary to develop a system modification that either dehumidifies the air before it reaches the evaporator or that can dry the evaporator coil even at the absence of gravity. While the first solution can be tested terrestrially, the latter needs a microgravity experiment for verification.

Open questions

- Can a system be modified such that liquid entering the compressor cannot occur?

- Can the system start-up independently of where the liquid refrigerant is located?

- Can a compressor be oillubricated independent of gravity?

- How can an evaporator be drained in microgravity after water condensed from air and accumulated on it?

- Will the heat transfer or the pressure drop of the two phase flow change significantly at the absence of gravity?

- Can a VCC operate independent of gravity?

\section{Needed Experiment}

Verify system modification against artificially created thread of liquid entering the compressor. System start-up with different locations of liquid refrigerant.

Testing of compressor performance at any possible orientation.
a) Verification of system that dehumidifies air before evaporator

b) Verification of gravity independent water draining. Heat exchanger or system testing in microgravity.

Testing of cycle at any possible orientation.

Table 2. Overview of open questions and needed experiments regarding operation of vapor compression systems in microgravity.

\section{Criticality of Experiments for Development of a Robust VCC for Microgravity}

The experiments listed in Table 2 are ranked according to their importance on the path of developing a robust VCC for microgravity. With the compressor being the only component in the system that could be damaged (by too much liquid coming into the compression chamber), a compressor protection system appears to be the most important modification and subject of testing. However, to further raise the TRL of VCC in microgravity, start-up and steadystate testing is also essential. The importance of gravity independent oil-lubrication in the compressor becomes unimportant if an oil-free compressor is selected. Nevertheless, oil-lubricated 
compression can significantly increase the efficiency and the life time. The importance of the gravity independent dehumidification system depends on the operating conditions, the duty time and the humidity of the air. The heat transfer and pressure drop will most likely change to some extent, but research on two-phase flow showed that flow boiling and flow condensation also occurs in microgravity and still has appreciable heat transfer coefficients. The gravity independent operation of the cycle is an interesting subject, but directly connected to performance penalties due to hydraulic losses and not necessary for a robust operation in microgravity.

\section{MATCHING OF TESTING POSSIBILITIES AND NEEDED EXPERIMENTS}

After introducing the five available testing platforms and six needed experiments, there is a total of 30 possible combinations, where each combination is an experiment defined by a goal and a platform. Figure 1 shows this information in a graphic representation with the five testing platforms on the left hand side and the six needed experiments on the right hand side. A solid connecting arrow means that the testing possibility supports the needed experiment well, while a dashed line means that there is a merit in performing the experiment but also some limitation. If no arrow is drawn, the testing platform is not applicable to the needed experiment. Table 3 lists all 30 possible experiments and also the suitability as indicated by the arrow style in Figure 1. Additionally, a short reasoning for the suitability of the experiment is provided.

\section{Suggested Path Forward}

Figure 1 presents on the left hand side the testing possibilities in order of increasing expenses and lead time. The inclinable test stand with four matching experiments is a strong candidate to start research, especially because two of the possible experiments have a high criticality for mission success. Following work on inclined testing, a parabolic flight experiment would be useful for a thorough test of the developed compressor protection system. A vapor compression cycle operating successfully on the alternating 0 and $1.8 \mathrm{~g}$ of a parabolic flight for about 30 parabolas, will considerably increase the TRL. The possible experiments on a sounding rocket are independent from the inclined testing and the parabolic flight and could be pursued in parallel to such work. A small scale vapor compression system on a sounding rocket has enough time to reach steady-state operation and thereby provide unique data covering the transient and steady-state operation of the cycle. In the same experiment, the heat exchanger effectiveness in microgravity can be analyzed, too. However, an experiment on a sounding rocket is available only at high cost and the test rig must fit in a relatively small enclosure. Testing on an orbital spacecraft is obviously very desirable due to the long available testing time. The cycle could be operated in steady-state at a variety of operating points and a system start-up could be tested multiple times. Another valuable merit is that the extended testing would allow to validate a theoretical model of the effects of microgravity. However, testing on an orbital spacecraft cannot cover all the open questions and 
therefore does not remove the need of utilizing other testing possibilities.

Match Suitability

1-A Good

By inclining the test stand, a worst case scenario can be created and the compressor protection system can be tested.

1-B Good By inclining the system, the liquid phase of the refrigerant can be artificially placed at different locations in the cycle.

1-C Good By inclining the stand and measuring the compressor performance, gravity independence of the oil-lubrication can be verified.

1-D,E N/A The inclinable test stand does not lend itself to specific microgravity testing.

1-F Good By inclining the stand and measuring certain state points, the gravity independence of the system can be verified.

$2-$

A,B, $\quad$ N/A

C,D,

E,F

3-A Good

If positioned specifically, the alternating $0 \mathrm{~g}$ and $1.8 \mathrm{~g}$ will extensively test the reliability of the compressor protection during operation.

3-B N/A The parabolic flight imposes chaotic locations of the refrigerant, so the testing could not be done in a targeted way.

Table 3. Overview of available matches and their suitability as well as comments. 


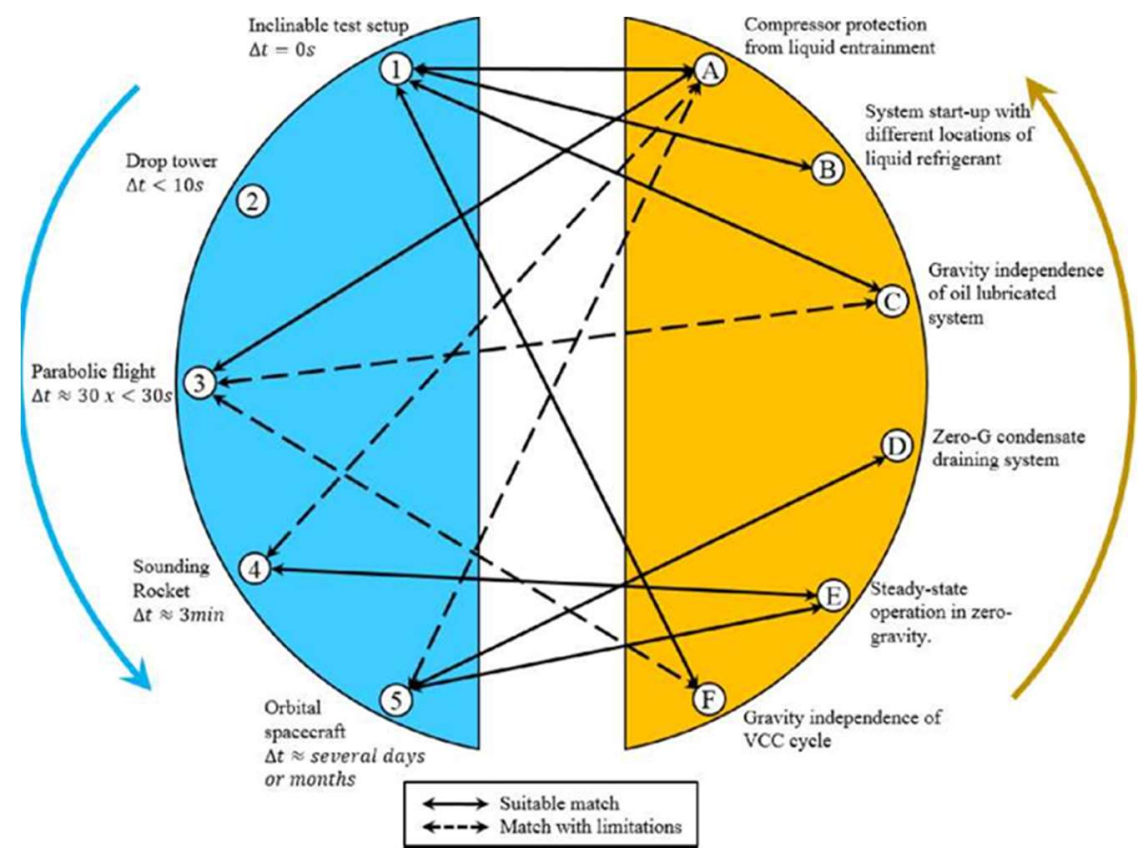

3-D N/A The time constant of the draining process would be longer than the microgravity duration.

3-C,F Limited The alternating $0 \mathrm{~g}$ and $1.8 \mathrm{~g}$ is a good test for gravity dependence. However, the system would have to be gravity independent up to $1.8 \mathrm{~g}$ to allow a positive result.

3-E N/A The microgravity duration is too short to achieve steadystate operation.

4-A Limited In microgravity, the compressor protection system may not be triggered.

4-B N/A The liquid refrigerant could not be controlled to be at different locations in the cycle during startup.

4-C,D N/A The microgravity testing platform could not verify gravity independence.

4-D N/A The time constant of the draining process would be too slow and the available space might be too small.

4-E Good The sounding rocket allows enough time for a small system to reach a steady state.

5-A Limited In microgravity, the compressor protection system may not be triggered.

5-B N/A In microgravity, the liquid in the system cannot be controlled to be at different locations in the cycle.

N/A The gravity independence cannot be verified in

5-C,F microgravity.

5-D Good The system could be tested well.

5-E Good The system could be tested well.

\section{CONCLUSIONS}

Vapor compression cycles in microgravity are desirable but have not been tested systematically in the past such that the knowledge about issues due to the absence of gravity is still small. The most critical problem appears to be liquid 
entering the compression chamber because it could lead to damage of the compressor. While steady-state operation in microgravity as an experiment is not essential, it would increase the TRL considerably. Gravity independence of the cycle is of low importance, but gravity independence of the oil-lubrication is important, if high compressor efficiencies become a dominant objective. Past experiments regarding vapor compression cycles in microgravity have only been conducted on orbital spacecraft, although less expensive testing possibilities are available, and some of them are even more suitable to verify robust operation than testing in a microgravity environment. While long term testing on an orbital spacecraft should be prepared, inclination testing, parabolic flights and sounding rockets all offer important experiments to be performed and should therefore be pursued.

\section{ACKNOWLEDGEMENTS}

The authors would like to thank Air Squared Inc., in particular Stephen L. Caskey and Bryce Shaffer, for funding the project that enabled this work and thank Michael K. Ewert for valuable advice and guidance.

\section{REFERENCES}

1. J.R. Blutt and S.E. Sadek, "A gravity independent vapor absorption refrigerator," Dynatech Corporation, Prepared under contract No. NASw-1372 NASA-CR-836, REPT.-695, Jul. 1967.

2. R.S. Johnston and L.F. Dietlein, "Biomedical Results from Skylab," National Aeronautics and Space Administration, NASA-SP-377, 1977.

3. S.M. Smith, S.R. Zwart, V. Kloeris, and M. Heer, Nutritional Biochemistry of Space Flight. Nova Science Publishers, 2009.

4. M. Cooper, M. Perchonok, and G.L. Douglas, "Initial assessment of the nutritional quality of the space food system over three years of ambient storage," npj Microgravity, vol. 3, no. 1, Dec. 2017.

5. D. Lipson, "STS-4 Postflight Final Report for the Vapor Phase Compression Refrigerator/Freezer," Technical Information Release DTO No. 467, Aug. 1982.

6. NASA, "Orbiter Refrigerator/Freezer Hardware Information Guide," Life Sciences Data Archive, 2018. [Online]. Available: https://lsda.jsc.nasa.gov/Hardware/hardw/64.

7. NASA, "Life Sciences Laboratory Equipment Refrigerator/Freezer Hardware Information," Life Sciences Data Archive, 2018. [Online]. Available: https://lsda.jsc.nasa.gov/Hardware/hardw/18.

8. NASA, "Refrigerated Centrifuge (RC)," International Space Station, 31-Mar2019. [Online]. Available:

https://www.nasa.gov/mission_pages/station/research/experiments/explorer/Investi gation.html?\#id=629.

9. H. Zhang, M. M. Hasan, and I. Mudawar, "A Method for Assessing the Importance of Body Force on Flow Boiling CHF," Journal of Heat Transfer, vol. 126, no. 2, pp. 161-168, 2004. 
10. H. Zhang, I. Mudawar, and M. M. Hasan, "Flow boiling CHF in microgravity," International Journal of Heat and Mass Transfer, vol. 48, no. 15, pp. 3107-3118, Jul. 2005.

11. K. V. Paiva, M. B. H. Mantelli, and L. K. Slongo, "Experimental testing of mini heat pipes under microgravity conditions aboard a suborbital rocket," Aerospace Science and Technology, vol. 45, pp. 367-375, Sep. 2015.

12. ZARM, "The Bremen Drop Tower," ZARM, UniversitatBremen, General Information. [Online]. Available: https://www.zarm.uni-bremen.de/en/droptower/general-information.html.

13. European Space Agency, "ESA User Guide to Low Gravity Platforms," European Space Agency.

14. H. Lee, I. Mudawar, and M. M. Hasan, "Experimental and theoretical investigation of annular flow condensation in microgravity," International Journal of Heat and Mass Transfer, vol. 61, pp. 293-309, Jun. 2013.

15. E.B. Wagner, "New Shephard - Payloads," presented at the CSF Microgravity Symposium, Tempe, Arizona, 16-Sep-2019.

16. V.A. Thomas, N. S. Prasad, and C. Ananda Mohan Reddy, "Microgravity research platforms - A study," Current Science, vol. 79, no. 3, pp. 336-340, Aug. 2000 .

17. Authors of the this paper, "(Personal experience)."

18. "Zero-G Research Programs," Zero-G - the weightless experience. [Online]. Available: https://www.gozerog.com/index.cfm?fuseaction=Research_Programs. welcome.

19. J. deFiebre and M. Guzik, "Assembly and Integrated Systems Testing for the Flow Boiling and Condensation Experiment (FBCE)," presented at the Thermal \& Fluids Analysis Workshop, Hampton, VA, Aug-2019.

20. L.P.M. Brendel, X. Zhang, J.E. Braun, and E.A. Groll, "Modelling of a Vapor Compression Cycle Independent of Orientation and Gravity," presented at the The 9th International Conference on Compressors and Refrigeration. 